\title{
The Awareness-/Coordination- Support-System Paradox
}

\author{
Christoph Oemig, Tom Gross \\ Human-Computer Interaction Group, University of Bamberg, Germany
}

\begin{abstract}
Coordination and awareness have been topics in the area of Computer-Supported Cooperative Work $(\mathrm{CSCW})$ starting from day one. Effortless coordination has been a major goal ever since. However, while awareness research became quite popular, coordination got increasingly out of focus. Its concepts stayed rather theoretical lacking concrete steps towards the original goal due to the absence of appropriate measurement techniques and tools. There is even an on-going general dispute if discount usability techniques deliver valid results as compared to real-world field studies. This paper supports their validity and demonstrates how the Standardized Coordination Task Assessment (SCTA) technique is used to extend the mechanics of collaboration, a framework for discount usability evaluations, allowing awareness and coordination effort assessments. The results appeared not only to be reasonable but the experiment revealed an effect we named the awareness-/coordination-support system paradox.
\end{abstract}

\section{Introduction}

Coordination and awareness have been topics in the area of Computer-Supported Cooperative Work (CSCW) starting from day one. Since 1992 it is known that mutual awareness is a fundamental concept that facilitates coordination (Beaudouin-Lafon \& Karsenty 1992). The latter was originally conceived as part of the 3C Model constituting the three main areas of CSCW (Ellis et al. 1991): communication, coordination, and cooperation. "Effortless coordination" (Gross 2013) or at least reducing coordination efforts to a minimum has been a major goal ever since. However, trying to achieve this goal revealed three major problems: First, in the past decades coordination and coordination support got increasingly out of focus (though being the higher goal). Focussing on awareness only was more popular but produced rather problematic results over time (Schmidt 2002). Second, coordination and direct coordination support stayed rather theoretical besides a few exceptions, e.g., the Coordinator (Flores et al. 1988). Most efforts delivered mainly concepts like the well-known coordination theory (Malone \& Crowstone 1990) or the empirically based coordination mechanisms (Schmidt \& Simone 1996). Yet, the positive effects of 
mutual awareness and coordination based upon that remained hard to measure. This leads to the final third problem: the measurement techniques and tools. There is still an ongoing dispute on whether the evaluation of cooperative systems using discount techniques delivers valid results (Steves et al. 2001). As one of these the mechanics of collaboration (Gutwin \& Greenberg 2000) are a framework providing the necessary constructs but lacking a concrete measurement approach in the area of coordination and awareness. However, with the advent of the Standardized Coordination Task Assessment (SCTA) (Oemig \& Gross 2011) appeared a new discount technique especially facilitating distinct awareness support and coordination support efficiency measurements.

This paper demonstrates how we use the SCTA to fill these gaps by extending the mechanics of collaboration adding the concepts and means for awareness and coordination support assessments. This especially facilitates the incremental development of effective support systems. After taking a brief look at related work we introduce the necessary background concepts, assumptions, and the measurement approach. We describe how they were applied in an experiment which also revealed an effect we named the awareness-/coordinationsupport-system paradox. After presenting and discussing the results this paper concludes with possible future work.

\section{Related Work}

Our work was inspired by mainly two research areas: Human Factors and CSCW. The former provides assessment methods like the Situation Awareness Global Assessment Technique (SAGAT) (Endsley et al. 1998), which is one of the most famous in this area. It was developed to assess situation awareness (SA) of air traffic controllers. SAGAT uses freeze probes to query participants concerning their perception of the current situation. The system's displays are blanked and the simulation is suspended while subjects quickly answer questions about their current perceptions of the situation. The method uses a predefined awareness model that includes Level 1 (perception of data), Level 2 (comprehension of meaning) and Level 3 (projection of the near future).

In $\mathrm{CSCW}$ there is still an ongoing discussion about whether discount usability evaluation techniques deliver valid results. Steves et al. (2001) proved their effective role in the evaluation of cooperative systems. Their study showed that discount techniques (heuristics, checklists, etc.) discovered many issues also found by real users in real work situations-but at tremendously lower cost (effort and time). Gutwin \& Greenberg (2000) even suggest a conceptual framework that describes the mechanics of collaboration, i.e., "the low level actions and interactions that must be carried out to complete a task in a shared manner. These include communication, coordination, planning, monitoring, assistance, and protection" (p.116). Their hypothesis: some usability problems in cooperative systems are not inherently tied to the social context in which the system is used. They are rather a result of poor support for these mechanics. Their framework suggests three general measures: effectiveness, efficiency, and satisfaction. 


\section{The Measurement Extension Concept}

Our concept picks up where the mechanics of collaboration left off: coordination is part of the mechanics while efficiency is part of the measures. Yet, there is no defined way in the framework that combines the two in a concrete measurement approach. This paper demonstrates how this gap can be closed using the SCTA approach. This section provides the overall rationale explaining the necessary background concepts, assumptions and measurement approach.

\subsection{Background}

When talking about efforts concerning awareness and coordination we especially focus on efforts imposed on the human being. Figure 1 depicts effort areas, key concepts and support systems.

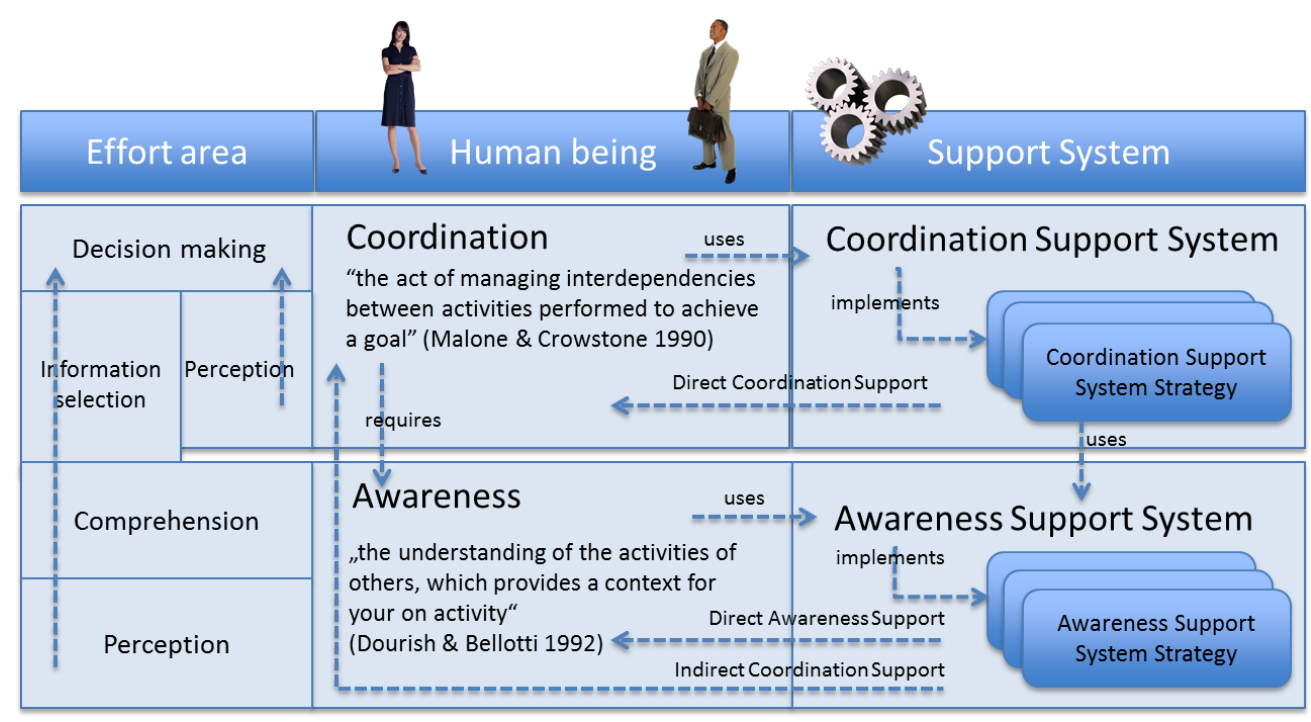

Figure 1: Overview of the key concepts, terms and their relationships.

We identified areas of effort resembling the above-mentioned three level model of situation awareness (Endsley 1995):

- Perception: continuous perception of relevant information from the environment

- Comprehension: processing information about the present and the past to arrange them in a common context

- Information selection: choosing the relevant information from the common context needed for decision making

- Decision-making: choosing a future options 
Having these areas in mind, decreasing the effort can be achieved only in a few ways: either by increasing efficiency of each single process or by moving efforts to substitute systems, i.e., by introducing specialized support systems. Examples are to reduce the information being perceived, formatting it for eased comprehension, or rule-based preparations of options for decision-making. Thus it becomes rather a question of splitting the overall effort between humans and support systems.

The parts of the system that take over these activities are the awareness support system and the coordination support system. Awareness support systems typically address the processes of perception (relevant to awareness) and comprehension. They let the user know who is around and what is going on. Awareness support can be realized in many different ways, one way of realization is called awareness support system strategy or short awareness strategy. Coordination support systems typically address the processes of perception (relevant to decision making), information selection and decision-making. Coordination support can be realized in different ways starting from providing all information that is needed up to nearly making the decision. The way it is realized is called coordination support system strategy or short coordination strategy.

\subsection{Assumptions}

Knowing the areas of effort it needs to be determined how this effort could be measured. For efforts regarding awareness on the side of the human being we use the following set of assumptions:

1. If I am aware about something, I can answer probe questions about it quickly and correctly.

2. The longer the answer takes, the more effort is involved.

3. The effort needed to answer the probe question is proportional to the regular effort during the task without a probe.

For efforts regarding coordination on the side of the human being, we use the following:

1. If I know my options, I can make correct and quick probe decisions.

2. The longer a decision takes, the more effort is involved.

3. The effort needed to make the probe decision is proportional to the regular effort during the task without a probe.

From these assumptions we are able to conclude the following: If options needed for my decision can be derived from that something I am aware of then awareness information facilitates coordination decisions (i.e., awareness facilitates coordination). Further we see that these options also can be derived from sources other than awareness information (e.g., from a coordination strategy). Using awareness support as a source thus becomes only one out of many other possibilities (cf. Figure 1). This finally leads to the following question: if there is a coordination strategy that actually does not involve awareness information, what happens to the awareness processes or the awareness support system? The next section describes the experiment used for verification. 


\subsection{Measurement Approach}

The Standardized Coordination Task Assessment (SCTA) (see Oemig \& Gross 2011 for details) is a measurement approach that applies a standard primary task (the counting of letters) to generate a certain awareness and coordination workload for a group of people. The group's goal is to count as many letters as possible. This allows the observation and assessment of awareness and coordination support strategies by measuring the efforts that remain imposed on the human being. The SCTA uses freeze probes to query participants about aspects of their awareness and their decision-making. Its basic assumptions are those described in the previous section. The tool SCTA Tracer (Oemig \& Gross 2012) uses questions concerning people and artefacts: Who counted a's? Were b's counted? Who may count c's next? What may count user A next? The application measures response times and whether the answer was correct or not. This corresponds to the efficiency measure of the mechanics of collaboration: quick and correct answers indicate smaller efforts and thus reasonable awareness or coordination support. The questions asked during the freeze probes are generated from the counting data the participants create during their task. Further advantages of the SCTA are its quantitative approach (number of correct answers, answering speed, performance (i.e. the number of counted letters)) and its simple reproduction of equivalent primary tasks, allowing large-scale measurements at very low cost.

\section{Experiment}

In order to demonstrate the extension of the mechanics of collaboration and to answer the open questions we designed an experiment contrasting a simple awareness support strategy and a simple coordination support strategy. Additionally, we used a control group with no support at all. In a first step the overall setup is described, a second step offers some of the experiment's results.

\subsection{Setup}

We defined three types of settings we wanted to measure. In a first setting we introduce a simple awareness support system. It implements a simple awareness strategy that notifies the user about what letter another user is about to count and about actual counting results (shown as "awareness display" in Figure 2). The second setting utilizes a simple coordination support strategy, we learned from earlier user feedback: the total number of letters is divided by the number of participants. Each user is assigned a respective fraction thereof. Once a letter is counted it is marked in the coordination support window (shown as "coordination display" in Figure 2). Thus the user does not need to know what the others are counting. The system basically coordinates the counting effort. Therefore, the coordination effort is expected to be at a minimum in this case. A third setting, the control group received no treatment at all. In order to avoid or minimize cofounding influences the experiment setting is selected by random for a single experiment run (i.e., the counting task). In the next step, the participants are assigned to the run by random as well. 


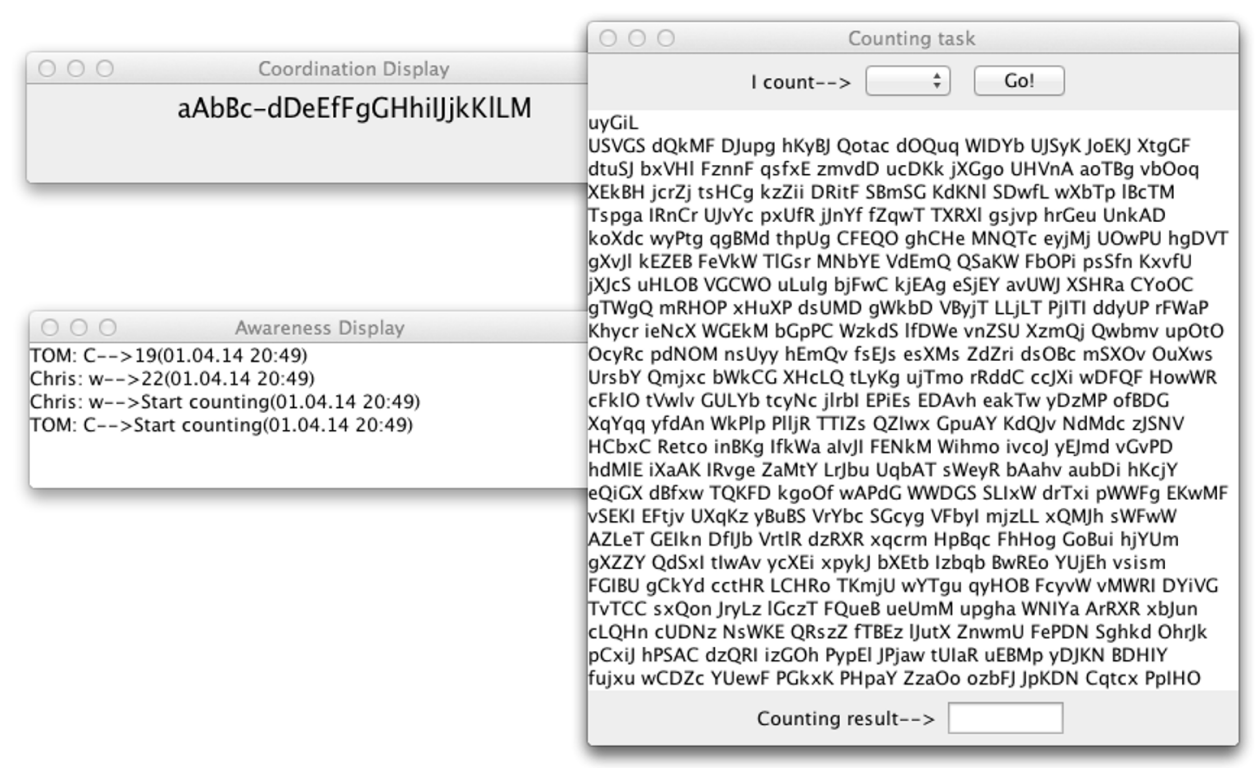

Figure 2: The SCTA counting task with awareness support and coordination support. This setup was not used in the experiment but it is to show all support options in one image. The experiment used either a simple awareness support, coordination support or no support at all.

There was a total number of 24 participants, $34 \%$ female and $66 \%$ male in a range of 17 to 40 years with the majority drawn from a computer science class (including the teacher) of a German High School. We only focused on dyads, i.e. from the total number of participants we always arranged groups with two members. They used individual computers separated by blinds so they could not see each other. They were not allowed to talk or to communicate by other means outside the provided tools. Since multiple counting tasks took place concurrently, participants did not even know with whom they were actually working (no real names were used). A counting task lasted 10 minutes interrupted by three freeze probes. These asked the participant two questions concerning group awareness, two questions concerning self awareness and two questions regarding coordination. Thus a total of 18 questions had to be answered by a participant during a run. Besides the performance (total number of counted letters) the coordination error rate (duplicate letters) was measured and recorded.

\subsection{Results}

This section briefly describes some of the most important results of this experiment. These are derived from the response time diagram (cf. Figure 3) and the success rates, performances and coordination errors shown in Table 1. 


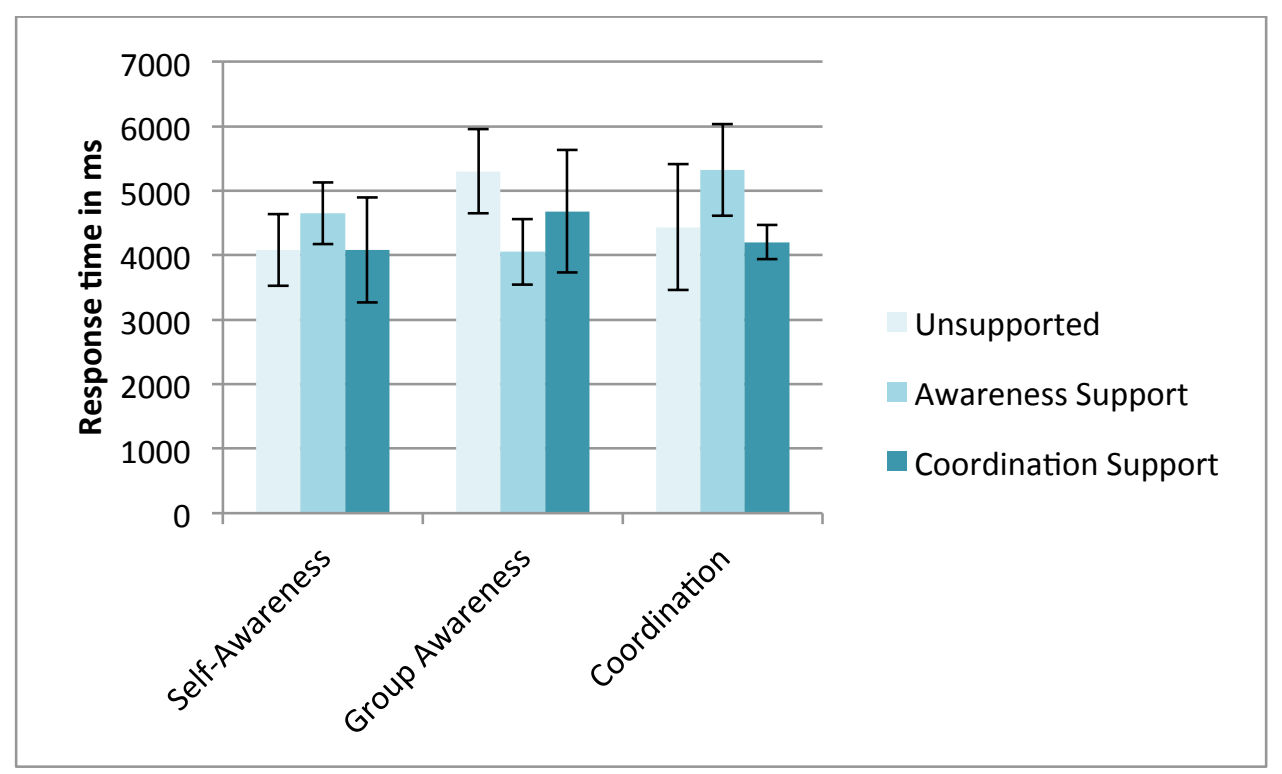

Figure 3: Mean response times per question area for each experiment type including standard error.

At first we looked for the most successful and fastest support for the SCTA categories self awareness, group awareness, and coordination. The experiment without any support type was the winner in the self awareness category (4s, 95\%) since participants probably had nothing else to really focus on. Not surprisingly the awareness support experiment type was the most successful and fastest $(4 \mathrm{~s}, 75 \%)$ in the category of group awareness. Last but not least the coordination support type experiment performed best $(4.2 \mathrm{~s}, 95 \%)$ in the coordination category. But the most interesting lies beyond the expected, which rather proves that the approach generally appears to work. Concerning our assumptions we could verify that awareness support facilitates coordination.

\begin{tabular}{|l|c|c|c|c|c|}
\hline \multirow{2}{*}{} & \multicolumn{2}{|c|}{ Success Rate (\%) } & \multicolumn{2}{c|}{$\begin{array}{l}\text { Performance } \\
\text { (Avg) }\end{array}$} & $\begin{array}{l}\text { Coordination } \\
\text { Errors (Avg) }\end{array}$ \\
\cline { 2 - 5 } & Self Aw. & Group Aw. & Coordination & 6 & 1 \\
\hline Unsupported & $\mathbf{9 5 \% ,}$ & $38 \%$, & $25 \%$ & 6 & 0 \\
\hline $\begin{array}{l}\text { Awareness } \\
\text { Support }\end{array}$ & $88 \%$, & $\mathbf{7 5 \% ,}$ & $70 \%$ & 7 & 0 \\
\hline $\begin{array}{l}\text { Coordination } \\
\text { Support }\end{array}$ & $75 \%$, & $63 \%$, & $\mathbf{9 5 \%}$ & 7 & \\
\hline
\end{tabular}

Table 1: Success rates (percentage of correct answers), performance (total number of letters counted), and coordination errors (number of letters counted multiple times).

However, it was not the fastest (5.2s) and created some errors (success rate $70 \%$ ), which might be due to its indirect nature in supporting coordination as mentioned in the concept. 
Only the direct coordination support performed better $(4.2 \mathrm{~s}, 95 \%)$, while the results for coordination of the unsupported type suggest that the participants were guessing $(4.5 \mathrm{~s}, 25 \%)$.

Furthermore, the data suggests that the coordination support does not require the user to have superior levels of self- and group awareness. Participants answered slower and increasingly wrong but overall performed best indicating that they do not really need this knowledge. We named this effect the awareness-/coordination-support system paradox: while awareness support facilitates coordination support the latter has not necessarily a need for the former. As expected the third setting (no support) shows the highest number of coordination errors. Yet, all types showed nearly the same overall performance. This is probably due to the large number of different countable letters (whole alphabet, small and capital letters) and the large quantity of each single letter. Therefore also the coordination errors are low requiring the reduction of the number of different letters to allow more coordination errors to occur.

\subsection{Discussion \& Consequences}

The experiment revealed some new insights and new problems regarding the goal of effortless coordination:

- The awareness-/coordination-support system paradox: this effect described above has the consequence that when developing a coordination support system it needs to be carefully reviewed which awareness information is still needed by the user. This adds another possibility to create more efficiency but, as the next insight shows, one needs to be careful.

- Effortless coordination's anti-social tendency: driving efficiency to the extreme may sacrifice social subtleties for its own purpose. Though performing best some participants mentioned that they did not like the coordination support experiment type. They could not see what their partner was doing or if he needed assistance. Reducing efforts to the minimum also contradicts general design principles of human-work. The consequence is not only to measure response times and errors but also include means to measure satisfaction. This is also mentioned as part of the mechanics of collaboration.

- Self awareness obviously needs no support: the unsupported experiment type actually removed all efforts concerning perception and comprehension from the user without offering a substitute. However, according to the measurement, participants performed even better in the category of self awareness suggesting that it is agnostic of any support system used. As a consequence self awareness does not need to be supported releasing screen estate and other resources for other information. Yet, it needs to be verified if this remains true for more complex settings.

The experiment further created insights regarding the used approach:

- The twofold letter problem: there were too many letters in too high quantities making it easy for the participants to avoid coordination errors. Further, this fact reduced the total number of letters counted since the participants spent longer times counting a single letter. This in turn reduced the effort needed to coordinate the situation. As a 
consequence future experiments will dramatically decrease the number of letters and their quantities.

- Don't know/don't care answer options: Another point mentioned by participants was that for some questions they would prefer an "I don't know" answer option instead of being forced to some other answer. This can also be derived from the results: the "I don't know" answers are those where there is a quick wrong or deliberate answer while producing an overall high performance. However, it needs to be discussed if an "I don't know" answer is sufficient or if an "I don't care" needs to be introduced as well since some of the knowledge is really not needed as the found paradox implies.

- Freeze probes killing counting results: Another aspect mentioned by participants is that the freeze probes prevented them sometimes from finishing their counting. Since there were only a few letters counted but those at high quantities this might have a large impact which can be avoided by smaller quantities for each letter and again reducing the number of letters overall.

\section{Conclusion}

In this paper we introduced a practical step towards the goal of effortless coordination. Extending on the mechanics of collaboration we used the SCTA technique to actually measure the performance, response times and success rates of different approaches trying to reduce the coordination efforts by introducing more efficiency or by moving them to support systems. In doing so we showed the effect we named the awareness-/coordination-support system paradox: while awareness support facilitates coordination support the latter has not necessarily a need for the former. It also turned out that there is a another yet disregarded constraint in the context of awareness and coordination support: the goal of effortless coordination must not sacrifice social subtleties for its own purpose.

However, this was actually just the beginning. Among many other possibilities a whole range of similar experiments can be done with group sizes beyond the dyad in one run adding more complexity, or with a longer duration beyond 10 minutes. There are many ways to refine the support systems and to repeat the measuring in an iterative approach including a comparison of results - a procedure actually intended by the authors of the mechanics of collaboration and of the SCTA.

\section{References}

Beaudouin-Lafon, M. and Karsenty (1992). A. Transparency and Awareness in a Real-time Groupware System. In Proceedings of The ACM Symposium on User Interface Software andTechnology UIST'92 (Nov. 15-18, Monterey, CA). ACM, N.Y., 1992. pp. 171-180.

Endsley, M.R. (1995). Toward a theory of situation awareness in dynamic systems. Human Factors $37(1), 32-64$.

Endsley, M.R., Selcon, S.J., Hardiman, T.D., Croft, D.G. (1998). A comparative evaluation of SAGAT and SART for evaluations of situation awareness. In Proceedings of the Human Factors and 
Ergonomics Society Annual Meeting, 1998, pp. 82.-86. Santa Monica, CA: Human Factors and Ergonomics Society

Ellis, C. A.; Gibbs, S. J. \& Rein, G. (1991). Groupware: Some Issues and Experiences. Commun. ACM, ACM, 1991, 34, 39-58

Flores, F.; Graves, M.; Hartfield, B. \& Winograd, T. (1988). Computer Systems and the Design of Organizational Interaction. ACM Trans. Inf. Syst., ACM, 1988, 6, 153-172

Gross, T. (2013). Supporting Effortless Coordination: 25 Years of Awareness Research. Computer Supported Cooperative Work: The Journal of Collaborative Computing, Springer-Verlag 22, 4-6 (Aug.-Dec. 2013). pp. 425-474. (SpringerLink, DOI:10.1007/s10606-013-9190-x).

Gutwin, C., Greenberg, S. (2000) The mechanics of collaboration developing low cost usability evaluation methods for shared workspaces. Proceedings of IEEE Workshop on Enabling Technologies - Infrastructure for Collaborative Enterprises, 116-121.

Malone, T. W. \& Crowston, K. (1990). What is Coordination Theory and How Can It Help Design Cooperative Work Systems? Proceedings of the 1990 ACM Conference on Computer-supported Cooperative Work, ACM, 1990, 357-370

Oemig, C. \& Gross, T. (2011). Illusive, Ineffective, Inefficient, Ideal: Standardized Coordination Task Assessments of Awareness Support. Mensch \& Computer 2011: überMEDIEN|ÜBERmorgen, Oldenbourg Verlag, 2011, 353-356

Oemig, C. \& Gross, T. (2012). SCTA Tracer: A Distributed Environment for Standardized Awareness Support Assessments. In Proceedings of the Twentieth Euromicro Conference on Parallel, Distributed, and Network-Based Processing - PDP 2012 (Feb. 15-17, Garching, Germany). IEEE Computer Society Press, Los Alamitos, 2012. pp. 52-56.

Schmidt, K. (2002). The Problem with 'Awareness': Introductory Remarks on 'Awareness in CSCW' Comput. Supported Coop. Work, Kluwer Academic Publishers, 2002, 11, 285-298

Schmidt, K. \& Simone, C. (1996). Coordination Mechanisms: Towards a Conceptual Foundation of CSCW Systems Design. Comput. Supported Coop. Work, Kluwer Academic Publishers, 1996, 5, $155-200$

Steves, M. P., Morse, E., Gutwin, C., und Greenberg, S. (2001). A comparison of usage evaluation and inspection methods for assessing groupware usability. In GROUP '01: Proceedings of the 2001 International ACM SIGGROUP Conference on Supporting Group Work, Seiten 125-134, New York, NY, USA. ACM Press

\section{Acknowledgements}

We like to thank all people participating in the initial setup of the assessment, especially our beta testers Michel Greiner and Nils Hottes, the participants of our experiment, and all reviewers for their valuable comments and suggestions on earlier versions of this work.

\section{Contact Information}

Prof. Dr. Tom Gross, E. tom.gross(at)uni-bamberg.de, T.(+49-951)863-3940

Christoph Oemig, MSc., coemig(at)acm.org 